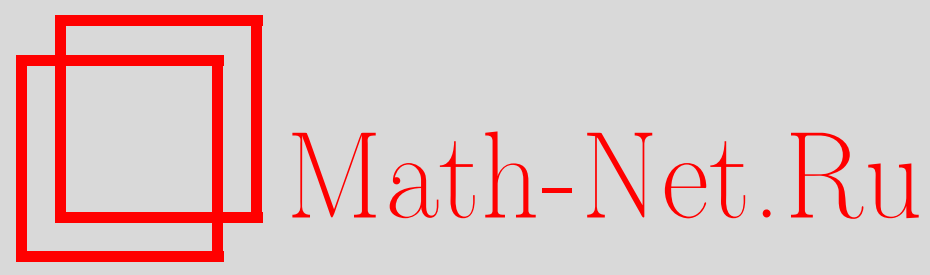

Е. И. Островский, Точная асимптотика интегралов Лапласа для негладких функций, Матем. заметки, 2003, том 73, выпуск 6, 886-890

DOI: https://doi.org/10.4213/mzm236

Использование Общероссийского математического портала Math-Net.Ru подразумевает, что вы прочитали и согласны с пользовательским соглашением http://www.mathnet.ru/rus/agreement

Параметры загрузки:

IP: 54.197 .130 .99

26 апреля 2023 г., 14:29:29

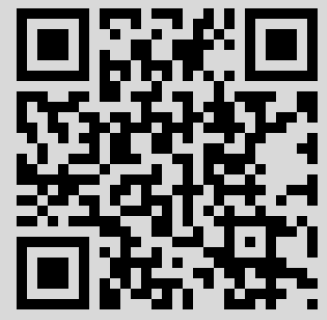




\section{ТОЧНАЯ АСИМПТОТИКА ИНТЕГРАЛОВ ЛАПЛАСА ДЛЯ НЕГЛАДКИХ ФУНКЦИЙ}

\section{Е.И. Островский}

В заметке вычисляется точная асимптотика с остаточным членом интегралов типа Лапласа в произвольном пространстве без каких-либо предположений о гладкости функций. В качестве частного случая вьводится классический результат. Рассмотрены примеры.

Библиография: 6 названий.

Введение и обозначения. Пусть $(X, B, \mu)$ - измеримое пространство с ограниченной мерой $\mu, \mu(X)<\infty ; S(x), g(x)$ - измеримые числовые функции, причем $S(x)$ существенно ограничена:

$$
M=\underset{x \in X}{\operatorname{vrai} \sup } S(x)<\infty,
$$

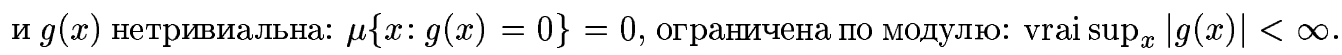
Положим

$$
I(\lambda)=\int_{X} g(x) \exp (\lambda S(x)) \mu(d x) .
$$

Здесь и далее $\lambda$ - "большой" числовой параметр: $\lambda \rightarrow \infty, \lambda \geqslant 2$. Интеграл в (1) понимается в смысле Лебега; из сформулированных условий вытекает его абсолютная сходимость.

В заметке вычисляется точная асимптотика, причем с остаточным членом $I(\lambda)$ в терминах, весьма близких к рассматривавшимися в [1].

Как следствие выводим классическую формулу Лапласа в различных ее модификациях и некоторые применения в теории вероятностей.

Никаких условий гладкости на исходные функции $g(\cdot), S(\cdot)$ (непрерывность, дифференцируемость и др.) мы не накладьваем; более того, на множестве $X$ непредполагается наличие какой-либо топологической структуры.

В работе [1] (см. также [2, с. 141]) выведены логарифмические асимптотики для $I(\lambda)$ и отмечены их применения в теории вероятностей. Автор хотел бы добавить, что в последнее время в этой науке отчетливо выявился интерес именно к точным асимптотикам. К примеру, в [3, с. 74-89] и далее выгисляется точная асимптотика распределений максимума гауссовских полей, в [4] - кратных стохастических интегралов, в [5] описаны и другие применения. 
Введем необходимые обозначения. Положим (частично по аналогии с [1], [2]) при $u \geqslant 0$

$$
\begin{gathered}
X(u)=\{x: x \in X, M>S(x)>M-u\}, \quad Y=\{x, x \in X, S(x)=M\}, \\
Z=\{x: x \in X, S(x)<M\}, \quad H(u)=\int_{X(u)} g(x) \mu(d x), \\
I_{0}(\lambda)=\int_{Z} g(x) \exp (\lambda S(x)) \mu(d x) .
\end{gathered}
$$

Отметим, что при $u \leqslant 0$ выполнено равенство $H(u)=0$.

Далее символы $C, C_{j}$ - конечные положительные константы, $\delta, \delta_{j}$ - малые положительные константы, $\delta=\delta_{1}<1 / 2, \delta_{j+1}<\delta_{j}, \Gamma(\cdot)$ - гамма функция,

$$
\begin{gathered}
K=\int_{Y} g(x) \mu(d x), \quad K_{1}(n)=\frac{\pi^{n / 2}}{\Gamma(1+n / 2)}, \quad K_{2}(n)=\frac{2 \pi^{n / 2}}{\Gamma(n / 2)} \\
K_{3}(n, v, \beta, \gamma)=v \beta^{-1} K_{2}(n) \Gamma\left(1+\frac{n-\gamma-1}{\beta}\right) \\
L(\lambda)=L(M, C, r, m ; \lambda)=C \Gamma(r+1) \exp (M \lambda) \lambda^{-r} \ln ^{m}(\lambda) \\
J(\lambda)=J(M, C, r, m ; \lambda)=\frac{I_{0}(\lambda)-L(\lambda)}{L(\lambda)}
\end{gathered}
$$

(Параметры $C, r, m$ будут введены чуть ниже.) Символ $A \sim B$ в том или ином предельном переходе $\lambda \rightarrow \infty, u \rightarrow 0$ и т.д. означает точную асимптотику: $\lim A / B=1$.

1. Формулировка основного результата. Поскольку $I(\lambda)=K \exp (M \lambda)+I_{0}(\lambda)$, то далее будем анализировать только интеграл $I_{0}(\lambda)$.

TEOPEMA. Ecлu $H(u) \sim C u^{r}|\ln u|^{m}, u \rightarrow 0+, r=$ const $>0, s=$ const, $C=$ const $\neq 0$, mo

$$
I_{0}(\lambda) \sim L(M, C, r, m ; \lambda)
$$

Пусть теперь при всех достаточно малых $u: u \in(0, \delta), \delta=$ const $\in(0,1 / 2), u \rightarrow 0+$ и при тех же возможных значениях $r, m, s, l$

$$
H(u)=C u^{r}|\ln u|^{m}+O\left(u^{s}|\ln u|^{l}\right), \quad s, l=\text { const },
$$

где предполагается, что $s>r$, либо $s=r, l<m$. Тогда при $m=0$

$$
J(\lambda)=O\left(\lambda^{r-s} \ln ^{l}(\lambda)\right)
$$

если же $m \neq 0$, но

a) $s>r$, то

$$
J(\lambda)=O\left(\ln ^{-1}(\lambda)\right) ;
$$

b) при $s=r$

$$
J(\lambda)=O\left(\ln ^{\max (m-1, l)-m}(\lambda)\right) .
$$


ЗАмЕчАниЕ 1. Как отмечалось в [1], условие на функцию $H(u)$ при соответствующих условиях может быть проверено с помощью результатов статьи [6].

2. Доказательство теоремы. Далее без ограничения общности будем считать $M=0$.

ЛЕммА. Пусть $\psi: \mathbb{R}^{1} \rightarrow \mathbb{R}^{1}$ - измеримая числовая функиия такая, что

$$
\int_{X}|g(x) \psi(S(x))| \mu(d x)<\infty .
$$

Tогда

$$
\int_{X} g(x) \psi(S(x)) \mu(d x)=\int_{0}^{\infty} \psi(u) d H(u)
$$

ДоКАЗАТЕЛЬСТво леммы проводится элементарными для теории меры методами. Обе части равенства (3) представляют собой линейные непрерьвные в норме

$$
\|\psi(\cdot)\|=\int_{X}|g(x)||\psi(S(x))| \mu(d x)
$$

функционалы от $\psi(\cdot)$. Поскольку обе части (3) совпадают для функций таких, что $\psi(S)=1$ при $S \in\left[C_{1}, C_{2}\right)$ и $\psi(S)=0$ при $S \notin\left[C_{1}, C_{2}\right]$, то лемма доказана.

ЗАмЕчАниЕ 2. Лемма может служить некоторой заменой формуле интегрирования по поверхностям уровня (равенство (3.24) из [2, с. 117], основанное на дифференциальной форме Лере-Гельфанда) в негладком случае.

Возврашаясь к доказываемой теореме, заметим, что из $(2)$, непрерьвности $H(u)$ в нуле и формулы интегрирования по частям следует

$$
I_{0}(\lambda)=\lambda \int_{0}^{\infty} \exp (-\lambda u) H(u) d u .
$$

Первое утверждение теоремы следует теперь из теоремы 1.7 монографии [2, с. 76]; докажем второе. Как и в [1], [2], выводим, что

$$
I_{0}(\lambda)=\lambda \int_{0}^{\delta} \exp (-\lambda u) H(u) d u+O(\exp (-\delta \lambda)) .
$$

Интеграл в правой части оценивается либо с помощью леммы Ватсона [2, с. 57-58]:

$$
\lambda \int_{0}^{\delta} u^{r} \exp (-\lambda u) d u=\Gamma(r+1) \lambda^{-r}+O\left(\exp \left(-\delta_{1} \lambda\right)\right)
$$

либо ее обобщений

$$
\lambda \int_{0}^{\delta} e^{-\lambda u} u^{r} \ln ^{m}(u) d u=L(\lambda)\left(1+O\left(\ln ^{-1}(\lambda)\right)\right.
$$

$[2$, c. 76$]$; вид остаточного члена в этих утверждениях различен. Вьполняя несложные вычисления и выделяя остаточньй член, приходим к утверждению теоремы. 


\section{3. Примеры.}

ПримеР 1. Рассмотрим классический метод Лапласа для кратных интегралов

$$
F(\lambda)=\int_{G} g(x) \exp (\lambda S(x)) d x, \quad x=\left(x_{1}, x_{2}, \ldots, x_{n}\right),
$$

где $G$ - ограниченная область в $\mathbb{R}^{n}$, содержащая начало координат, $g(x)$ - непрерывная в нуле ограниченная функция, $S(\cdot) \in C^{\infty}([G])$ и достигает единственного максимума в точке $x=0$. Без ограничения общности считаем, что $g(x)=1, S(x)<S(0)=0, x \neq 0$,

$$
\frac{\partial^{2} S}{\partial x_{i} \partial x_{j}}(0)=\Delta(i, j)=1, \quad i=j
$$

и $\Delta(i, j)=0$ в противном случае. Тогда

$$
\operatorname{mes}\{x: S(x) \leqslant u\} \sim \operatorname{mes}\left\{x: \sum_{i=1}^{n} x_{i}^{2} \leqslant u\right\} \sim K_{1}(n) u^{n / 2}, \quad u \rightarrow 0+,
$$

где mes - мера Лебега. Подставляя в (2), получаем классический результат [2, с. 122]. Разумеется, строгое доказательство возможно на основе теоремы Морса, согласно которой после гладкой замены переменньх с единичным якобианом в некоторой окрестности нуля $S(x)$ имеет в точности вид, указанный вьше. Аналогично рассматривается случай нескольких, граничных или вырожденных точек максимума. Таким образом, классический факт вытекает из нашего результата.

ПримеР 2. Видоизменяя предыдущий случай, возьмем

$$
S(x)=-|x|^{\beta}, \quad \beta=\text { const }>0,
$$

а в качестве $f(x)$ будем рассматривать функцию вида $f(x)=C_{2}|x|^{-\gamma},|x|=\sqrt{\sum_{i} x_{i}^{2}}$, $\gamma=$ const $\in(0, n)$. Тогда

$$
H(u) \sim \int_{x:|x|^{\beta}<u}|x|^{-\gamma} d x \sim C_{2} u^{(n-\gamma) / \beta},
$$

откуда согласно теореме

$$
I(\lambda) \sim C_{2} \Gamma\left(\frac{(n-\gamma)}{\beta}+1\right) \lambda^{(\gamma-n) / \beta}
$$

ПРимеР 3. Следующий пример фактически заимствован из [1], но мы находим точную асимптотику. Пусть

$$
S(x)=-\left|\sum_{i=1}^{n} x_{i}^{2 m(i)}-1\right|^{\beta}, \quad g(x)=1,
$$

$\beta=$ const $>0, m(i)$ - целые числа, большие 1 . Тогда $H(u) \sim A u^{1 / \beta}, A=$ const [1]. По формуле (2) имеем

$$
I(\lambda) \sim A \Gamma\left(1+\frac{1}{\beta}\right) \lambda^{-1 / \beta} .
$$

Формула (4) легко обобщается на случай любой невырожденной гладкой гиперповерхности. 
ПРимеР 4. Последний пример - из теории вероятностей. Пусть $\xi, \eta$ - одномерные случайные величины, определенные на вероятностном пространстве $(\Omega, B, \mathrm{P}), \Omega=\{\omega\}$, причем vrai $\sup _{\omega}|\eta(\omega)|<\infty, \operatorname{vrai}_{\sup _{\omega}} \xi(\omega)=M<\infty$. Обозначим

$$
I(\lambda)=\int_{\Omega} \eta \exp (\lambda \xi) \mathrm{P}(d \omega), \quad q=\mathrm{P}(\xi=M),
$$

или, на вероятностном языке, $I(\lambda)=\mathrm{E} \eta \exp (\lambda \xi), \mathrm{E}$ - математическое ожидание на вероятностном пространстве $(\Omega, B, \mathrm{P})$,

$$
H(u)=\int_{\omega: M>\xi>M-u} \eta(\omega) \mathrm{P}(d \omega)=\iint_{x: M>x>M-u} y F(d x, d y),
$$

$F\left(D_{1}, D_{2}\right)=\mathrm{P}\left(\xi \in D_{1}, \eta \in D_{2}\right)$ - совместное распределение $\xi, \eta$. Нас интересует асимптотика $I(\lambda), \lambda \rightarrow \infty$. (Случай, когда $M=\infty$, рассмотрен в [5, гл. 1].)

Пусть, как и ранее, $H(u) \sim C u^{r}|\ln u|^{m}, u \rightarrow 0+, C \neq 0$; тогда на основании теоремы имеем

$$
\begin{gathered}
I(\lambda)=K \exp (\lambda M)+I_{0}(\lambda), \\
I_{0}(\lambda) \sim C \Gamma(r+1) \exp (M \lambda) \lambda^{-r} \ln ^{m}(\lambda), \quad \lambda \rightarrow \infty .
\end{gathered}
$$

ЗАмЕчАниЕ 3. Предложенный метод непосредственно не обобщается на случай чисто мнимой “фазы” $S(x)$, поскольку для метода стационарной фазы существенна еще и гладкость подьнтегральных функций.

\section{СПИСОК ЦИТИРОВАННОЙ ЛИТЕРАТУРЫ}

[1] Маслов В.П., Федорюк М.В. Логарифмическая асимптотика интегралов Лапласа // Матем. заметки. 1981. Т. 30. С. 880-883.

[2] Федорюк М. В. Асимптотика. Интегралы и ряды. М.: Наука, 1987.

[3] Питербарг В.И. Асимптотические методы в теории гауссовских случайных процессов и полей. М.: МГУ, 1984.

[4] Островский Е. И. Точная асимптотика плотности распределения кратного стохастического интеграла // Проблемы передачи информации. 1992. Т. 61. №6. С. 817-824.

[5] Островский Е. И. Экспоненциальные оценки для случайных полей и их применения. Обнинск: ОИАТЭ, 1999.

[6] Attiyah M. F. Resolution of singularities and division of distributions // Comm. Pure Appl. Math. 1970. V. 23. №2. P. 145-150.

Институт индустриальной математики, г. Реховот, Израиль E-mail: ignat@102.net.il 